ministered with a MAD Nasal (Wolfe Tory Medical Inc, Salt Lake City, UT) mucosal atomizing device. Doses were administered in $1 / 6$ dose increments in alternating nares. Pain scores were recorded at $0,2,5$, and 10 minutes using a verbally administered numerical rating scale of 0 through 10 .

Results.-Data analysis was performed using results from 42 of the 46 patients: 5 pediatric and 37 adult. Four patients were excluded due to incomplete data. Thirty-four patients were initially treated on-slope and 8 patients were initially treated in the clinic. Average weight-based dosage for intranasal fentanyl was $1.4 \mu \mathrm{g} / \mathrm{kg}$ (95\% confidence interval [CI]: $1.3-1.5 \mu \mathrm{g} / \mathrm{kg} ; \mathrm{n}=42$ ). The mean baseline pain score for all patients was 8.2 (95\% CI: 7.7-8.7; $\mathrm{n}=42$ ). Pain scores were significantly reduced after treatment with fentanyl. Mean pain score reduction at 2 minutes was $-1.4(95 \% \mathrm{CI}$ : -2.0 to $-0.96 ; \mathrm{n}=41)$; at 5 minutes, $-2.8(95 \% \mathrm{CI}:-3.5$ to -2.1 ; $\mathrm{n}=42)$; at 10 minutes, $-2.8(95 \%$ CI: -3.7 to $-1.9 ; \mathrm{n}=29)$. No significant complications were noted.

Conclusion.-Intranasal fentanyl provides effective analgesia in acutely injured patients and is a good option for patients in whom immediate intravenous access is complicated by environmental, anatomic, or resource limitations. The potential application for search-and-rescue and other austere medicine situations is widespread.

\author{
A. Robb McLean, MD \\ Darren A. Braude, MD \\ Cameron S. Crandall, MD \\ Albuquerque, NM, USA
}

Timothy D. Peterson, MD Taos Ski Valley, NM, USA

\section{Comparison of 4 Hemostatic Agents, CELOX-A, ChitoFlex, WoundStat, and Combat Gauze, Versus Standard Gauze Dressing in Control of Extremity Hemorrhage in a Limited Access Swine Model of Penetrating Combat Trauma}

Objectives.-Exsanguination from extremity wounds remains the leading etiology of preventable combat death. We conducted a randomized, prospective, unblinded trial to investigate the efficacy of the most commonly used hemostatic agents in a model of severe vascular injury with mixed high-pressure arterial and venous bleeding in a small, linear tract wound that was designed to replicate a penetrating injury from a projectile where the bleeding site cannot be directly visualized.

Methods.-A complex groin injury with transection of the femoral vessels through a $3-\mathrm{cm}$ entrance wound, followed by 45 seconds of uncontrolled hemorrhage, was created in 80 swine prior to randomization to 5 groups. Group 1 used standard gauze; group 2 CELOX; group 3 Chitoflex; group 4 Combat Gauze; and group 5 WoundStat. Each agent was applied with 5 minutes of manual pressure prior to resuscitation. Hemodynamic parameters were recorded over 180 minutes. Primary endpoints included initial hemostasis and incidence of rebleeding. Secondary endpoints included a composite index of adverse events (the 2 primary endpoints and mortality).
Results.-Composite adverse events consisting of mortality, posttreatment hemorrhage, and failure of initial hemostasis were compared between treatment groups using single degree of freedom $\gamma^{2}$ analysis. Chi-square values were Yates-corrected to obtain conservative tests of statistical significance. Four of $16(25 \%)$ CELOX-A, 10 of $16(62.5 \%)$ ChitoFlex, 6 of 16 (37.5\%) Combat Gauze, 11 of $16(68.8 \%)$ WoundStat, and 7 of $16(43.8 \%)$ standard dressing subjects suffered from adverse events. A significant difference was found between the agents CELOX-A and WoundStat with respect to composite adverse events $(P=.0335)$.

Conclusions.-Our study demonstrated that CELOX-A was superior to WoundStat in controlling hemorrhage in smaller limited access wounds. There were no statistically significant differences in gauze products when compared to CELOX-A. Therefore, standard gauze and adequate wound packing were found to perform equally as well as advanced hemostatic agent products in controlling hemorrhage in smaller, linear tract wounds without direct visualization of the bleeding vessels.

Lanny F. Littlejohn, MD John J. Devlin, MD

Sara S. Kircher, BS, RLAT Robert Lueken, MD Michael R. Melia, MD

Andrew S. Johnson, MD Portsmouth, VA, USA

\section{Internet Accuracy Regarding Snake Envenomation Prehospital Care}

Snake envenomation is a cause of significant morbidity and mortality throughout the world. Historically, the threat posed from the bite of a venomous snake led to the use of numerous treatment modalities of variable efficacy, many based on traditional practice rather than sound scientific knowledge. Research has proven many of these unfounded practices to be ineffective and actually harmful.

With increasing use of the Internet for health information by both clinicians and patients, there is concern that improper treatment modalities regarding snake envenomation prehospital care are being perpetuated. To investigate this hypothesis, 2 major search engines were used to review 48 websites regarding 7 prehospital treatment options for snake envenomation (suction, immobilization, cryotherapy, heat, electric shock, incision, and removal of constrictive devices). Websites were evaluated for their quality using the Health on the Net (HON) seal and Journal of American Medical Association (JAMA) benchmarks.

Of the 48 websites reviewed, 26 contained inaccurate recommendations, and the remaining 22 websites were accurate regarding all topics addressed. Among the websites reviewed, improper treatment recommendations included: suction (14); ice/cryotherapy (6); incision (4); and electric shock (1). Five websites (fda.gov, MedlinePlus.gov, umm.edu) that met all 4 JAMA benchmarks and the HON seal included 3 improper treatment recommendations. The 5 websites that met none of 
the JAMA benchmarks nor included the HON seal included only 2 improper treatment measures.

This study highlights the variety of misinformation available on the internet regarding prehospital care of snake envenomation and the unreliability of the HON seal and JAMA benchmarks as markers of accuracy. As the internet becomes an increasing source of medical information for both the public and clinicians, the importance of accurate websites becomes imperative. Clinicians and patients should be aware of the high variability of internet information regarding snake bite prehospital care.

Sarah J. Barker, MD

Nathan P. Charlton, MD

Christopher P. Holstege, MD

Charlottesville, VA, USA

\section{Do Waterborne Coliform Bacteria Increase in Sierra Nevada Mountain Wilderness Area Lakes and Streams During Drought Periods?}

Objectives.-Humans may acquire waterborne infections in remote wilderness environments. We hypothesize the risk of waterborne infection increases during drought years.

Methods.-Water was collected from wilderness area lakes and streams in the Sierra Nevada Mountains of California during summer 2007 and 2008, and results compared to a previously published data set from this laboratory. Precipitation during water years 2007 and 2008 was $45 \%$ and 55\% of normal, respectively. Collection locations matched those from prior years in Yosemite, Kings Canyon-Sequoia (SEKI) National Parks and Forest Service Wilderness. Land use was categorized as predominate impact from either: 1) wildlife (W), 2) day-hike (DH), 3) backpackers (BP) only, or 4) pack animals (PA). Water was collected in sterile tubes and Millipore coliform samplers and analyzed at the University of California, Davis research laboratory, where bacteria were harvested, then subjected to analysis using standardized techniques. Coliforms were used as a marker of infection risk; samples showing $>100$ CFU/100 mL were considered positive. Statistical analysis to compare among site categories was performed using Fisher exact test.

Results.-A total of 168 sites were sampled (84 each year) among the 3 types of wilderness. Prevalence of coliforms is listed by area and land use (\% positive, raw number positive sites/total sites):

\begin{tabular}{llccc}
\hline & Wild & Day-hike & Backpack & Pack animal \\
Location & $(W)$ & $(D H)$ & $(B P)$ & $(P A)$ \\
\hline
\end{tabular}

Yosemite $\quad 35 \%(5 / 13) \quad 10 \%(2 / 10) \quad 20 \%(5 / 23) \quad 78 \%(18 / 23)$

$\begin{array}{lllll}\text { SEKI } & 0 \%(0 / 14) & 14 \%(1 / 7) & 17 \%(2 / 12) & 68 \%(23 / 34)\end{array}$

Forest wild $20 \%(1 / 5) \quad 50 \%(1 / 2) \quad 17 \%(1 / 6) \quad 95 \%(18 / 19)$

$\begin{array}{lllll}\text { Totals } & 19 \%(6 / 32) & 21 \%(4 / 19) & 20 \%(8 / 41) & 75 \%(59 / 76)\end{array}$

No difference was found comparing 2007 to 2008; coliform prevalence was significantly higher in PA use areas when compared to either $\mathrm{W}, \mathrm{DH}$, or $\mathrm{BP}$ areas $(\mathrm{P} \leq .05)$. Results were also compared to coliform prevalence from 364 samplings collected during 5 nondrought years (2002-2006) previously reported as follows: W 9\%, DH 12\%, BP 18\%, and PA $63 \%$. Coliform prevalence was higher in the drought years compared to nondrought, in $\mathrm{W}, \mathrm{DH}$, and PA areas but only significant in PA areas $(59 / 76$ vs $70 / 111 ; P<.05)$.

Conclusion.-During drought years, wilderness water coliform prevalence was significantly increased compared to nondrought years in those areas used by pack animals.

Robert W. Derlet, MD

John R. Richards, MD

Sacramento, CA, USA

Kemal A. Ger, PhD

Davis, CA, USA

\section{A Novel Technology for Assessing Cerebral Blood Flow at Altitude}

Introduction.-Most current assessments of cerebral hemodynamics at altitude rely on transcranial dopplers (TCDs). This technology does not monitor cerebral blood flow (CBF), per se, but rather blood flow velocity. Equating CBF to blood flow velocity is predicated on multiple assumptions that remain unproven at altitude, such as constant diameter of mediumsized arteries. An increase in middle cerebral artery velocity as measured by TCD is interpreted as evidence of vasospasm within the context of subarachnoid hemorrhage, for example, but vasodilation within the context of ascent to altitude. Current techniques to directly assess CBF, such as Xenon CT (XeCT), are either too cumbersome or too expensive to allow serial measurements to assess the temporal dynamics of changes in CBF during ascent to high altitude.

We present a human feasibility study of a technique, previously validated in animal models, that allows measurement of $\mathrm{CBF}$ that is uniquely suited to investigation of blood flow changes at altitude.

Methods.-Near infrared spectroscopy coupled with intravenous injection of indocyanin green dye (ICG-NIRS) was used to measure a CBF index in 3 patients undergoing laparoscopic surgery to assess feasibility of this technique in humans.

Results.-Two of the 3 patients exhibited an increase in $\mathrm{CBF}$ index of $40 \%$ and $59 \%$, during insufflation of $\mathrm{CO}_{2}$. The third patient exhibited a paradoxical decrease in $\mathrm{CBF}$ index of $39 \%$.

Conclusions.-The combination of ICG-NIRS and determination of CBF index is a promising, minimally invasive, portable and straightforward technique to provide repeated quantitative information regarding changes in CBF. This method is well suited to use in the alpine environment. Further refinements in technique and equipment, as well as formal validation studies in humans, are needed to increase the utility of ICGNIRS.

Matthew R. Sanborn, MD W. Andrew Kofke, MD Philadelphia, PA, USA 